\title{
Communication \\ Retrotransposons Down- and Up-Regulation in Aging Somatic Tissues
}

\author{
Giorgia Giordani $^{1}$ (D), Valeria Cavaliere ${ }^{1}$, Giuseppe Gargiulo ${ }^{1}$, Giovanna Lattanzi ${ }^{2,3}$ (D) \\ and Davide Andrenacci $2,3, *$ (D)
}

1 Dipartimento di Farmacia e Biotecnologie, Alma Mater Studiorum Università di Bologna, 40126 Bologna, Italy; giorgia.giordani3@unibo.it (G.G.); valeria.cavaliere@unibo.it (V.C.); giuseppe.gargiulo@unibo.it (G.G.)

2 CNR Institute of Molecular Genetics “Luigi-Luca Cavalli-Sforza” Unit of Bologna, 40136 Bologna, Italy; giovanna.lattanzi@cnr.it

3 IRCCS Istituto Ortopedico Rizzoli, 40136 Bologna, Italy

* Correspondence: dandrena@area.bo.cnr.it

Citation: Giordani, G.; Cavaliere, V.; Gargiulo, G.; Lattanzi, G.; Andrenacci, D. Retrotransposons Down- and Up-Regulation in Aging Somatic Tissues. Cells 2022, 11, 79. https://doi.org/10.3390/cells 11010079

Academic Editors: Laura Fanti and Ali Hamiche

Received: 3 November 2021

Accepted: 26 December 2021

Published: 28 December 2021

Publisher's Note: MDPI stays neutral with regard to jurisdictional claims in published maps and institutional affiliations.

Copyright: (c) 2021 by the authors. Licensee MDPI, Basel, Switzerland. This article is an open access article distributed under the terms and conditions of the Creative Commons Attribution (CC BY) license (https:// creativecommons.org/licenses/by/ $4.0 /)$.

\begin{abstract}
The transposon theory of aging hypothesizes the activation of transposable elements (TEs) in somatic tissues with age, leading to a shortening of the lifespan. It is thought that TE activation in aging produces an increase in DNA double-strand breaks, contributing to genome instability and promoting the activation of inflammatory responses. To investigate how TE regulation changes in somatic tissues during aging, we analyzed the expression of some TEs, as well as a source of small RNAs that specifically silence the analyzed TEs; the Drosophila cluster named flamenco. We found significant variations in the expression levels of all the analyzed TEs during aging, with a trend toward reduction in middle-aged adults and reactivation in older individuals that suggests dynamic regulation during the lifespan.
\end{abstract}

Keywords: longevity; transposons; gypsy; ZAM; Idefix

\section{Introduction}

Transposable elements (TEs) are DNA sequences able to proliferate in genomes by mechanisms that allow these elements to transpose into new sites. Among TEs, retrotransposons are characterized by a mechanism of replication through reverse transcription of an RNA intermediate. Retrotransposons are, in turn, classified as either long terminal repeats (LTR) retrotransposons or non-LTR retrotransposons. In Drosophila, a specific group of retrotransposons are repressed in the somatic cells of adult ovaries by the activity of a small RNA cluster named flamenco [1]. Like other small RNA clusters, flamenco is constituted of TE fragments, which are expressed in the form of a precursor long non-coding RNA. The transcript is then processed to produce Piwi-interacting RNAs (piRNAs) that silence the regulated TEs. In somatic tissues outside the ovary, endogenous small interfering RNAs (endo-siRNAs) mapping to the flamenco locus were also found [2], suggesting its involvement in the regulation of TEs outside the reproductive organs, as well. Among the mobile elements controlled by flamenco there are at least three LTR-retrotransposons: gypsy, ZAM and Idefix [3-5]. It is known that the ability to silence these mobile elements is variable and there are Drosophila lines that are permissive for their mobilization and lines that are non-permissive [3,5]. A well-characterized line permissive for the mobilization of ZAM and Idefix (Rev line) displays large deletions that remove ZAM and Idefix sequences in the flamenco locus [6]. This allows ZAM and Idefix expression in the follicular epithelium and in somatic tissues outside the ovary throughout Drosophila development (in embryos, larvae and adult flies) [7]. Permissive lines for gypsy mobilization have also been characterized, allowing gypsy activation in follicular cells and in the head tissues [8-10]. Regulation of TEs by flamenco involves the PIWI protein in the reproductive apparatus, and is PIWI independent in the somatic tissues, suggesting different molecular mechanisms of repression [7]. 
Although these mechanisms have been investigated, nothing is known about what happens to the expression of small RNA clusters in aging, and whether there is a correlation with changes in the expression levels of the regulated TEs. Increased mobilization of some TEs in somatic tissues during aging has been considered to be a cause of lifespan-shortening, and the observation of this phenomenon is at the base of the "transposon theory of aging" [11,12]. Reduction of constitutive heterochromatin during lifespan [13] is considered to be the cause of the derepression of a number of TEs, which are mainly located in heterochromatin regions [14]. Their transcriptional activation, which is associated to an increase of transposition events, increases the production of DNA double-strand breaks (DSBs), contributing to genome instability and the activation of inflammatory responses [15-17].

Considering the role of the small RNA clusters in TE silencing, we wondered what happens to the expression of flamenco and some TEs that are potentially regulated by this cluster in aging, using allele combinations that produce flies able or unable to repress gypsy. Analyses were performed comparing these expressions in the head tissues of young, middleaged and old females. We found a substantial repression of most of the analyzed sequences in middle-aged flies, and increased expression in old flies. Expression of retrotransposons and flamenco were different when comparing young flies able to repress gypsy with those unable to repress gypsy, while they were very similar in old flies. This suggests differing capabilities to repress TE at different points during the lifespan. Furthermore, flies with a higher level of gypsy expression showed a small reduction in longevity with respect to individuals demonstrating lower gypsy expression. This finding supports a possible correlation between gypsy activation and longevity.

\section{Materials and Methods}

\subsection{Drosophila Stocks}

Drosophila stocks were kept at $25^{\circ} \mathrm{C}$ on a standard cornmeal/yeast medium. Old flies were collected for each genotype and used for the experiments. To obtain middle-aged and old flies, young individuals were collected in freshly prepared food vials that were kept horizontally. Food vials were changed every three days. The following strains were used: Canton S (wild type), flam ${ }^{A}, w^{I R 6 R e v I I 7}, D f(1)$ l11/FM7c B flam ${ }^{\text {FM7 }} ;$ P $\{$ gypsy-lacZ.p12\} [8] and flam $^{B G 02658}$. Df(1)l11 deletion includes eor, flam, and wap genes [18]. Since the nonpermissive flies carry the $\operatorname{Bar}(B)$ dominant mutation, these flies were distinguished from the permissive ones on the base of the dominant Bar phenotype of the eyes.

\subsection{Quantitative RT-PCR}

Total RNA was extracted by crushing in TRI Reagent (Sigma-Aldrich, St. Louis, MO, USA) five ovaries (three days old), or ten female heads of different selected ages. The removal of genomic DNA contamination and synthesis of the complementary cDNA was performed by HiScript III 1st Strand cDNA Synthesis Kit (+gDNA wiper) (Vazyme, Nanjing, China), according to the manufacturer's protocol. Gene expression analysis was performed using the Power SYBR Green PCR master mix (Thermo Fisher Scientific, Waltham, MA, USA) in 96-well Piko PCR Plates (Thermo Fisher Scientific, Waltham, MA, USA) using the PikoRel 96 Real-Time PCR System (Thermo Fisher Scientific, Waltham, MA, USA), according to the manufacturer's suggested procedure. To determine target specificity, dissociation curve analysis was performed. RNA levels were normalized to the internal standard gene $R p 49$. The $\Delta \Delta \mathrm{Ct}$ method was used to measure the expression levels. The calibrator used for each experiment is reported in the figure legend. Three biological replicates were used for each experiment, and the average was calculated. All reported errors are the standard deviation of the mean. For quantitative PCR (qPCR) primers see Supplemental Material, Table S1 [19].

\subsection{Lifespan Analysis}

Mated females were used in the experiments. Animals were maintained in the climate chamber at $25^{\circ} \mathrm{C}$ on a standard cornmeal/yeast medium in a $12 \mathrm{~h}$ light, $12 \mathrm{~h}$ dark regime. 
Three Drosophila vials containing 20 females per vial were used for each genotype. Animals were relocated to a fresh medium every three days. Dead flies were counted daily.

\subsection{Statistical Analyses}

For the comparison of two groups with the same age and different genotype, P-values were calculated using an unpaired $t$-test. Where multiple genotypes were compared, a one-way ANOVA was performed, applying a Tukey post hoc test. Where multiple ages and genotypes were compared, a two-way ANOVA was performed, and the results are reported in the figure legends. For the comparison of two groups with different ages, a post hoc analysis was performed, applying the LSD test.

Survival analyses for the lifespan curves were performed using the Kaplan-Meier method with the Log Rank test. The calculation of the average lifespan was performed with this formula: (day $1 \times$ dead numbers of this day + day $2 \times$ dead numbers of this day $+\ldots$ day $n \times$ dead numbers of this day)/total dead numbers. Standard deviation was also calculated.

IBM SPSS software version 25 was used for all of the statistical analyses.

\section{Results}

\subsection{Gypsy, ZAM and Idefix Expression Is under Flamenco Regulation in Head Tissues}

We analyzed the expression of gypsy, ZAM and Idefix, comparing the Canton $S$ (CS) wild type strain with the flam $^{A}$ strain, which carries a flamenco permissive allele for gypsy mobilization [19], and the $w^{I R 6 R e v I I 7}$ strain, which carries a flamenco permissive allele for Idefix mobilization $[20,21]$. The CS strain showed low expression levels for all three analyzed retrotransposons (Figure 1A). On the other hand, the $\operatorname{flam}^{A}$ strain showed a high gypsy expression level and low ZAM and Idefix levels, while $w^{\text {IR6RevII7 }}$ strain showed a high Idefix expression level and low gypsy and ZAM expression levels (Figure 1A). These data confirm that gypsy and Idefix are upregulated in the head when there is a specific permissive allele of flamenco, similarly to what happens in ovaries. Using a couple of primers designed to amplify the $5^{\prime}$ coding region of flamenco (flam1) (Figure S1), we found very similar transcriptional levels of flamenco in the three genetic backgrounds (Figure 1B). Similar results were obtained using a second couple of primers (flam5) designed downstream of flam1 (Figures 1B and S1). To further support the hypothesis that flamenco is involved in the regulation of the analyzed retrotransposons in the head's somatic tissues, we analyzed the effect of the $\operatorname{flam}^{B G}$ allele. It is reported that this allele, which derives from the P element insertional mutation in the $5^{\prime}$ of the flamenco locus (Figure S1), is permissive for gypsy and ZAM expression in the follicular epithelium [4]. RT-qPCR experiments have confirmed that gypsy is strongly upregulated, while flamenco is downregulated in the ovary [1]. Expression analysis in flam $^{B G}$ head tissues confirmed that gypsy and ZAM are significantly upregulated compared to CS (Figure 1A), while flamenco is significantly downregulated (Figure 1B). All these data are in agreement with the hypothesis that flamenco has a role in the regulation of the three retrotransposons in the head tissue. 
A
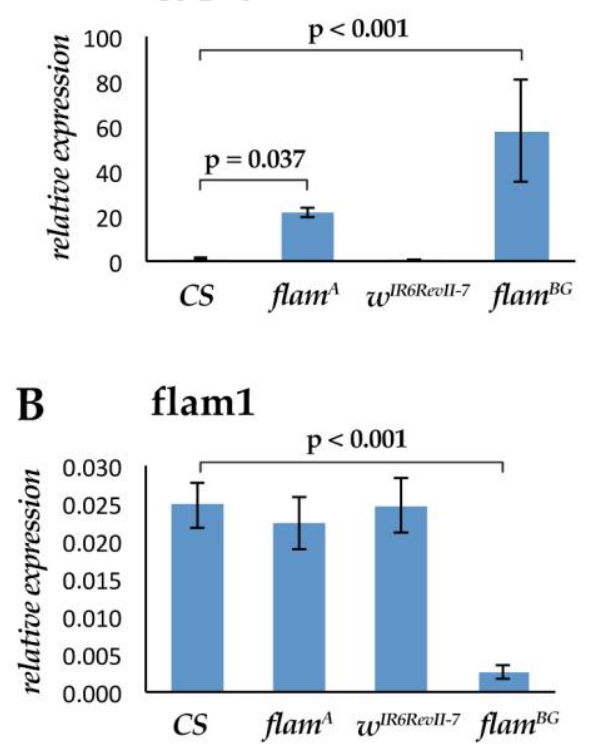

ZAM

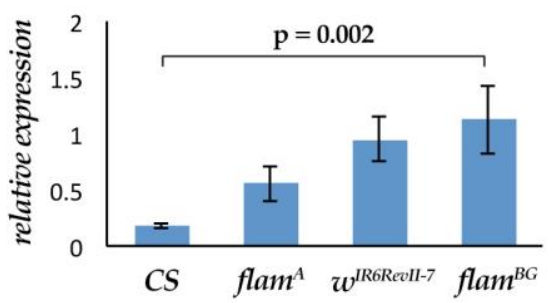

flam5

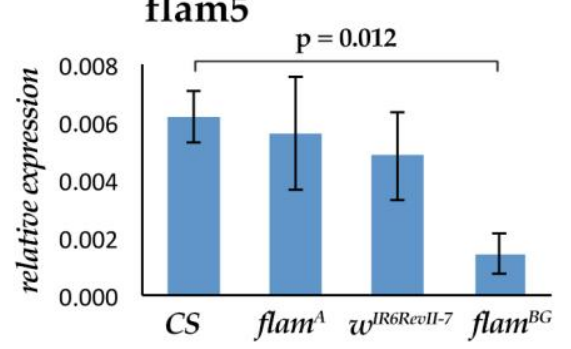

Idefix

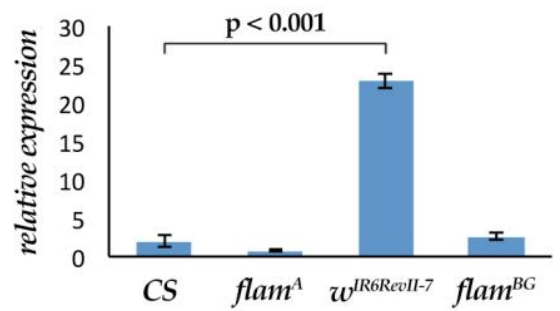

Figure 1. gypsy, ZAM and Idefix retrotransposons are under the regulation of flamenco in head somatic tissues. (A,B) Gene expression analysis in RNAs isolated from female head tissues from Canton $S(C S)$, flam $^{A} /$ flam $^{A}, w^{I R 6 R e v I I 7} / w^{I R 6 R e v I I 7}$ and flam $^{B G} /$ flam $^{B G}$ strains. The fold changes indicate the expression levels relative to calibrator (gypsy in CS). (A) Expression analysis of gypsy, ZAM and Idefix. (B) Expression analysis of flamenco. Shown are average levels $(n=3)$, and error bars indicate SD. Significance was determined by one-way ANOVA with Tukey post hoc test.

\subsection{Aging Effects on the Expression of Flamenco and Flamenco Regulated Retrotransposons}

We decided to analyze the expression of these three TEs and of flamenco in female heads during aging. Analyses were performed on Day 1 (young), Day 30 (middle-aged) and Day 50 (old) flies. We also tested whether a different ability to silence at least one retrotransposon could affect the regulation of TEs in aging. To this end, we analyzed the expression of the TEs and flamenco in two groups of flies derived from the same genetic cross and carrying a different flamenco allelic combination in a similar genetic background (Figure 2A). We used the flam $^{A}$ strain because the high gypsy expression level found in this strain is associated to high retrotransposon activity [19]. To obtain flies that were nonpermissive (NP) and permissive (P) for gypsy mobilization, we crossed flam $^{A}$ males $(\mathrm{P})$ with $D f(1) l 11 / f l a m^{F M 7}$ females [19]. $D f(1) l 11$ deletion encompasses the flamenco locus, while the flam $^{F M 7}$ is a dominant gypsy non-permissive allele carried by an $F M 7 c$ balancer (Figure 2A). From this cross, flam $^{A} / D f(1) l 11$ (P) flies were obtained, which carried a gypsy permissive allele in the hemizygous condition, and flam $^{A} /$ flam $^{F M 7}$ (NP) flies, with a permissive and a dominant non-permissive allele. As expected, we found that, in adult ovaries, gypsy expression was significantly higher in P than in NP flies (Figure 2B). A significantly higher expression level of gypsy in P flies was also found in the head tissues of young adult females (Figure 2C). These data confirm that flamenco is involved in gypsy regulation in the head, even if the effect of flamenco on the regulation of gypsy in these somatic tissues seems to have a minor impact. Comparing the expression of the three retrotransposons regulated by flamenco in young individuals of the two genetic backgrounds, we found that all showed slightly lower expression levels in the NP genetic background (Figure 2C-E). Furthermore, flamenco expression was two times higher in NP versus P flies (Figure 2F) due to the presence of a single copy of the flamenco gene in the latter. 
A

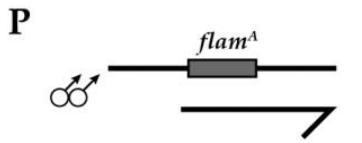

F1

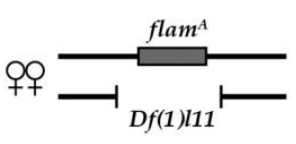

permissive
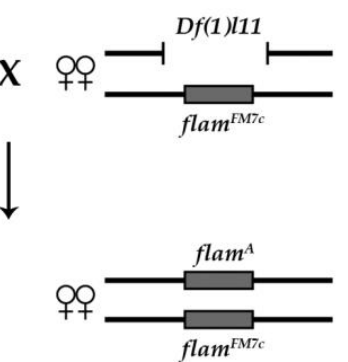

non permissive
C

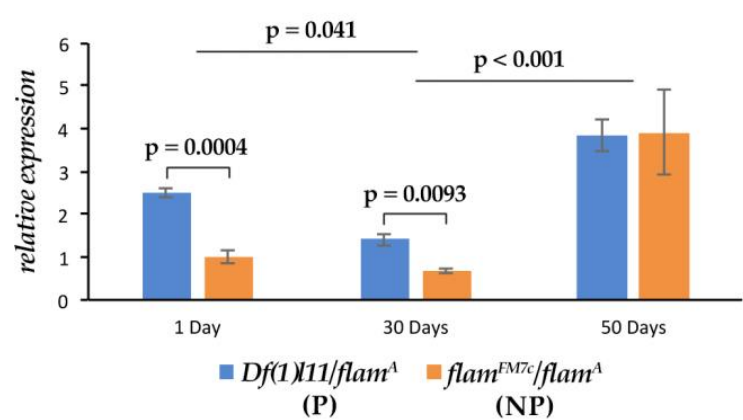

E

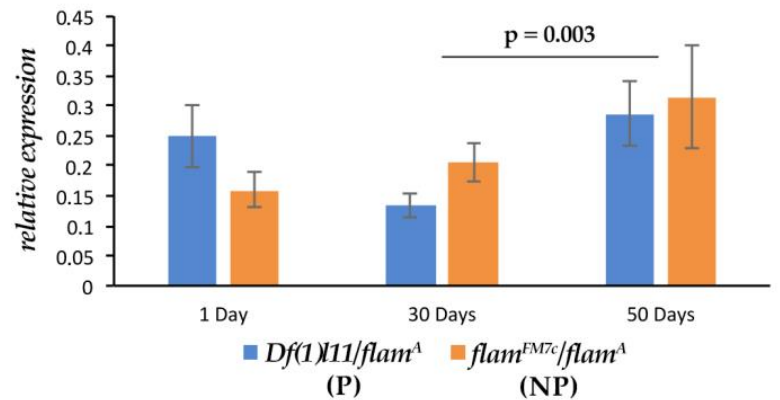

G

flam5 (head)

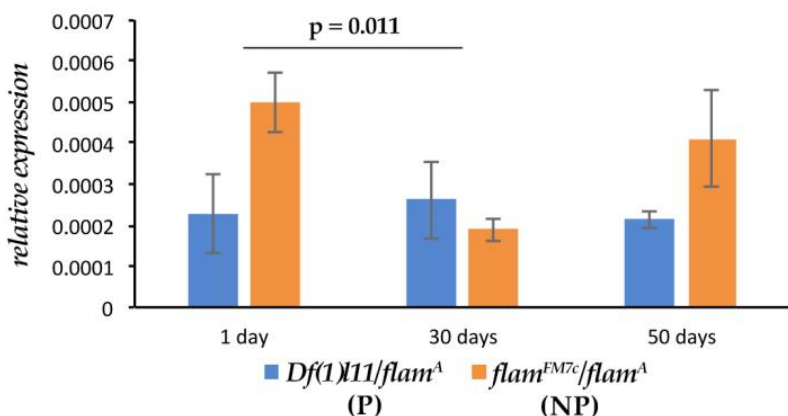

B gypsy (ovary)

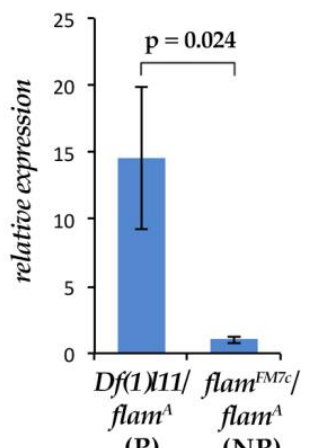

D

ZAM (head)

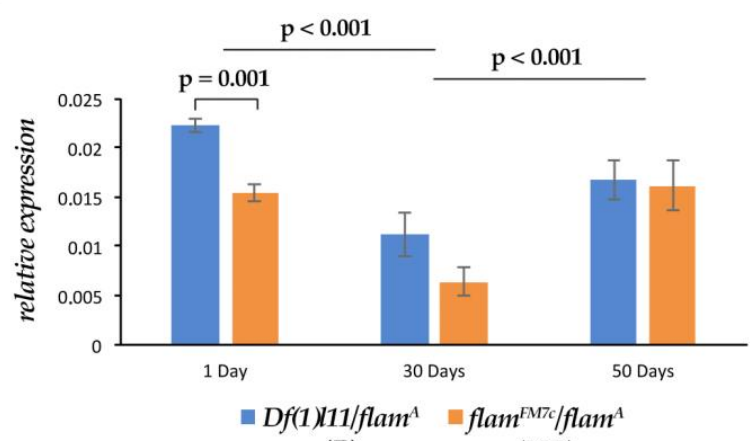

(P) $\quad(\mathrm{NP})$

F

flam1 (head)

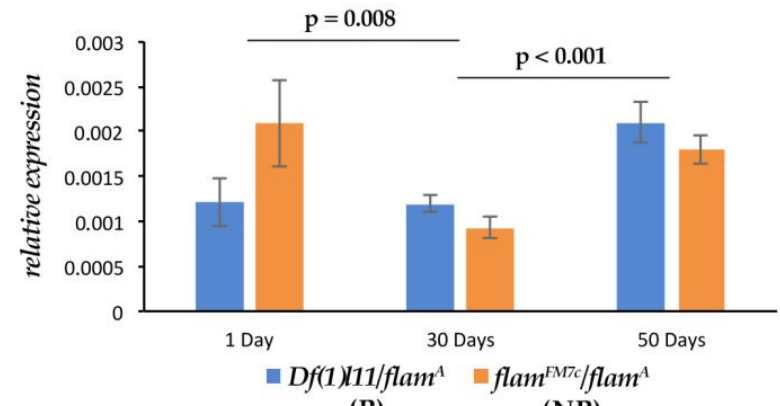

(P)

H

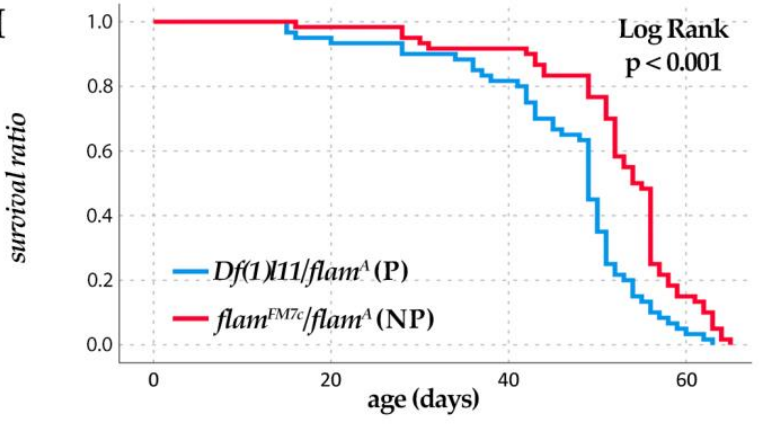

Figure 2. Analysis of the expression of the selected retrotransposons and of flamenco in aging. (A) Schematic representation of the genetic cross performed to obtain individuals permissive and non-permissive for the activation of gypsy. (B) Analysis of the expression of gypsy in RNAs isolated from 3-days-old ovaries of flam $^{A} / \mathrm{Df}(1) l 11$ and flam $^{A} / \mathrm{flam}^{\mathrm{FM} 7}$ adult females. The fold changes indicate the expression levels relative to calibrator $\left(\right.$ flam $^{A} /$ flam $\left.^{F M 7}\right)(\mathbf{C}-\mathbf{F})$ Gene expression analysis 
in RNAs isolated from head tissues from flam $^{A} / D f(1) l 11$ and flam $^{A} /$ flam $^{F M 7}$ adult females on Day 1 (young), Day 30 (middle-aged) and Day 50 (old). The fold changes indicate the expression levels relative to calibrator (gypsy in 1 day-old flam $^{A} /$ flam $^{F M 7}$ ) (C) gypsy expression. A two-way ANOVA reveals a significant effect of genotype $(p=0.015)$ and of age $(p=0.001)$. (D) ZAM expression. A two-way ANOVA reveals a significant effect of genotype $(p=0.002)$ and of age $(p=0.001)$. (E) Idefix expression. A two-way ANOVA reveals a significant effect of age $(p=0.009)$. (F) flam1 expression. A two-way ANOVA reveals a significant effect of genotype/age $(p=0.011)$ and of age $(p=0.001)$. (G) flam5 expression. A two-way ANOVA reveals a significant effect of genotype/age $(p=0.007)$, of genotype $(p=0.004)$ and of age $(p=0.034)$. Shown are average levels $(n=3)$, and error bars indicate SD. Comparison of two groups with different ages was done by applying a LSD post hoc analysis. Pairwise comparisons between genotypes of the same age were done using a two tails unpaired $t$-test. (H) Kaplan-Meier survival curves of $D f(1) l 11 /$ flam $^{A}$ and flam $^{F M 7 C} /$ flam $^{A}$ adult females.

We then analyzed variations in the expression levels of the three TEs and of flamenco in aging. We found that the significant difference in the expression levels of gypsy between $\mathrm{P}$ and NP was maintained in middle-aged flies (Figure 2C). A significant reduction in the expression levels was observed for gypsy, ZAM and flamenco in the transition from young to middle-aged flies, while Idefix shows an opposite pattern for P and NP (Figure 2C-G). In the transition from middle-aged to old flies, the expression levels of all the analyzed TEs were significantly upregulated, becoming quite similar in P and NP flies (Figure 2C-E). A similar activation effect was also observed for flamenco, even if small differences were observed when comparing the expression of the two regions analyzed (Figure 2F,G). These data show that the differences in the expression levels of gypsy and ZAM found at Day 1 and Day 30 between P and NP disappear in old individuals.

We found longevity differences between $\mathrm{P}$ and NP flies, which were manifested after the first 30 days of life, with P flies showing a slightly higher mortality rate (Figure $2 \mathrm{H}$ ). The average lifespan of P flies was $46.22 \pm 1.39$ days while that of NP flies was $52.25 \pm 1.2$ days.

\section{Discussion}

The reported data suggest that the expression of TEs can change with a down- and up-regulation trend during aging. In fact, after the first half of the Drosophila adult life (Day 30), we found a significant down-regulation for two of the retrotransposons analyzed (gypsy and ZAM) in both the genetic backgrounds and for Idefix in the P genetic background (Figure 2). In the transition from middle-aged to old flies (from Day 30 to Day 50), we found a significant increase in the expression levels of all of the retrotransposons analyzed. Retrotransposon down- and up-regulation has been also described for the Drosophila Copia element in adult testis, in which it is down regulated in 12-15-day-old tissues with respect to three-day-old tissues and again upregulated at 24-27 days [22].

The enhanced ability of NP somatic tissues to repress gypsy and ZAM expression in young individuals is no longer found in old individuals. Aging seems to smooth out differences in the regulation of retrotransposons between P and NP somatic tissues. The expression of flamenco, one of the main regulators of these TEs, changes over time in a similar way to that of the retrotransposons under its control. The significant reduction levels of transcript in middle-aged individuals could depend on mechanisms of cosuppression, as previously described for gypsy and flamenco [19], which could explain the concomitant repression of gypsy, ZAM and flamenco. Cosuppression, which has been described to act at the post transcriptional level [19], is triggered by gypsy proliferation as a consequence of the high retrotransposon expression level (Figure 3). This mechanism suppresses the deleterious effect of gypsy proliferation, which would inevitably lead to higher expression levels and higher transposition rates. Endo-siRNAs, sense and antisense to TE sequences, have been found in somatic tissues [2], and their increase could explain the increased silencing of both retrotransposons and flamenco. Activation in old age of both retrotransposons and flamenco could be explained by the accumulation of unprocessed 
flamenco transcripts, and a reduction in the production of small RNAs that serve to silence the target TEs (Figure 3). Furthermore, high expression levels could also be sustained by the derepression of retrotransposon copies that could be located in heterochromatin regions, in response to the reduction of constitutive heterochromatin that is found in old individuals [14].

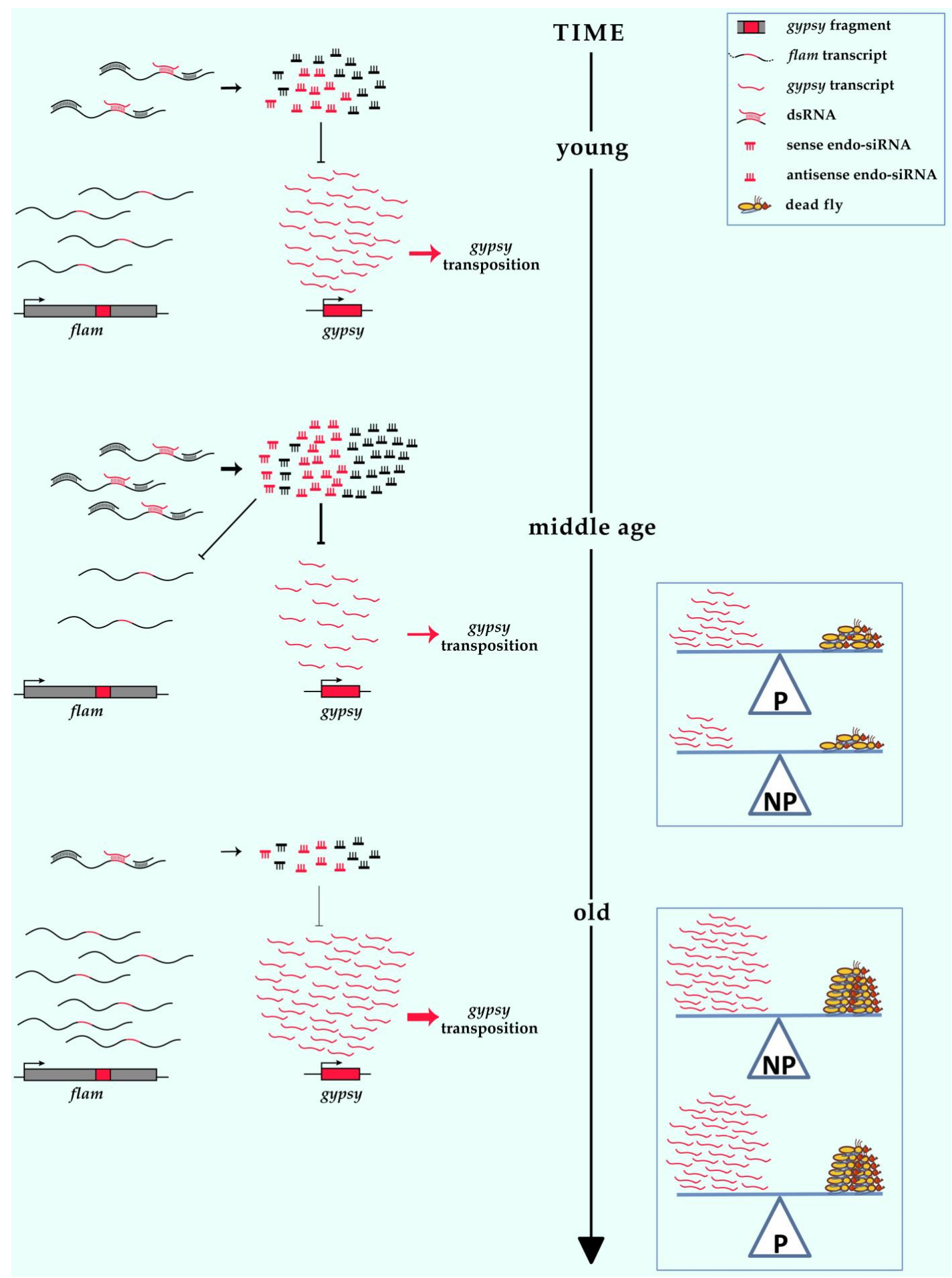

Figure 3. A speculative model for gypsy regulation during the aging process. flamenco is highly enriched in antisense TE sequences, comprising gypsy fragments. The flamenco primary transcript can form double strand RNAs (dsRNAs) with gypsy sense transcripts that serve as substrates for the production of endo-siRNAs specific for the silencing of gypsy. In young flies, gypsy expression level 
depends on its transcriptional rate and on post-transcriptional silencing induced by specific endosiRNAs (gypsy-antisense). In middle age, reduction in gypsy and flamenco expression can be explained by an increased production of endo-siRNAs (sense and antisense) specific for the silencing of gypsy and gypsy fragments that are part of the flamenco transcript (cosuppression). In old flies, gypsy and flamenco appeared derepressed, and this can be explained by a reduction of silencing, probably due to a reduction in the amount of endo-siRNAs (sense and antisense). Similar mechanisms can be hypothesized to explain down- and up- regulation of ZAM and Idefix in aging. Right boxes: a legend is represented in the upper box; correlation between the abundance of gypsy transcription and mortality in P(permissive) and NP (non-permissive) flies in middle aged individuals (middle age box) and old individuals (old box).

The difference in the average lifespan between gypsy permissive and non-permissive flies (Figure 2H) supports the idea that gypsy activation could be involved in the shortening of lifespan (Figure 3). Significantly, a shorter lifespan has been described in Ago2 mutations, which disrupt the somatic TE control mechanism and increase expression of the retrotransposons gypsy and $R 2$ in brain [23]. It has also been described that lines exhibiting earlier gypsy transposition events showed a shorter lifespan, while those with later transposition events showed a longer lifespan [17]. All of these results are in agreement with the hypothesis of the involvement of TE activation in reducing the lifespan.

As a whole, our study suggests that the investigation of a possible role for the distribution of TEs in the genome and of their activation state during aging could shed light on physiological and pathogenetic differences among aging individuals. The study of the role of small RNA clusters in regulating TE expression in aging could help to identify the causes of expression variation of some TEs in aging.

Supplementary Materials: The following are available online at https:/ /www.mdpi.com/article/10 .3390 / cells11010079/s1, Figure S1: Schematic representation of the flamenco locus and the regions where the specific primer pairs map; Table S1: Primer sequences used in this study.

Author Contributions: D.A. designed and supervised the study. G.G. (Giorgia Giordani), V.C. and D.A performed the experiments. D.A., V.C., G.G. (Giorgia Giordani) and G.G. (Giuseppe Gargiulo) analyzed and interpreted the data. D.A. wrote the manuscript with input from V.C., G.G. (Giuseppe Gargiulo), G.G. (Giorgia Giordani) and G.L., G.L., G.G. (Giuseppe Gargiulo) and V.C. acquired the funding. All authors have read and agreed to the published version of the manuscript.

Funding: G.L. was funded by EU E-RARE 2017 project “TREAT-HGPS”, AIProSaB 3/2019 project "Cross-talk between inflammatory cytokines and progerin", G.G. (Giuseppe Gargiulo) and V.C. were funded by a research grant from the University of Bologna, RFO2019.

Institutional Review Board Statement: Not applicable.

Informed Consent Statement: Not applicable.

Data Availability Statement: Not applicable.

Acknowledgments: We thank the Bloomington Drosophila Stock Center and Aurelio Valmori for technical support and William Blalock for his editorial assistance in preparing this manuscript.

Conflicts of Interest: The authors declare no conflict of interest.

\section{References}

1. Brennecke, J.; Aravin, A.A.; Stark, A.; Dus, M.; Kellis, M.; Sachidanandam, R.; Hannon, G.J. Discrete Small RNA-Generating Loci as Master Regulators of Transposon Activity in Drosophila. Cell 2007, 128, 1089-1103. [CrossRef]

2. Ghildiyal, M.; Seitz, H.; Horwich, M.D.; Li, C.; Du, T.; Lee, S.; Xu, J.; Kittler, E.L.; Zapp, M.L.; Weng, Z.; et al. Endogenous siRNAs derived from transposons and mRNAs in Drosophila somatic cells. Science 2008, 320, 1077-1081. [CrossRef] [PubMed]

3. Desset, S.; Conte, C.; Dimitri, P.; Calco, V.; Dastugue, B.; Vaury, C. Mobilization of two retroelements, ZAM and Idefix, in a novel unstable line of Drosophila melanogaster. Mol. Biol. Evol. 1999, 16, 54-66. [CrossRef] [PubMed] 
4. Mével-Ninio, M.; Pelisson, A.; Kinder, J.; Campos, A.R.; Bucheton, A. The flamenco locus controls the gypsy and ZAM retroviruses and is required for Drosophila oogenesis. Genetics 2007, 175, 1615-1624. [CrossRef] [PubMed]

5. Pélisson, A.; Song, S.U.; Prud'homme, N.; Smith, P.A.; Bucheton, A.; Corces, V.G. Gypsy transposition correlates with the production of a retroviral envelope-like protein under the tissue-specific control of the Drosophila flamenco gene. Embo J. 1994, 13, 4401-4411. [CrossRef]

6. Zanni, V.; Eymery, A.; Coiffet, M.; Zytnicki, M.; Luyten, I.; Quesneville, H.; Vaury, C.; Jensen, S. Distribution, evolution, and diversity of retrotransposons at the flamenco locus reflect the regulatory properties of piRNA clusters. Proc. Natl. Acad. Sci. USA 2013, 110, 19842-19847. [CrossRef]

7. Desset, S.; Buchon, N.; Meignin, C.; Coiffet, M.; Vaury, C. In Drosophila melanogaster the COM locus directs the somatic silencing of two retrotransposons through both Piwi-dependent and -independent pathways. PLoS ONE 2008, 3, e1526. [CrossRef]

8. Cavaliere, V.; Lattanzi, G.; Andrenacci, D. Silencing of Euchromatic Transposable Elements as a Consequence of Nuclear Lamina Dysfunction. Cells 2020, 9, 625. [CrossRef]

9. Pélisson, A.; Sarot, E.; Payen-Groschêne, G.; Bucheton, A. A novel repeat-associated small interfering RNA-mediated silencing pathway downregulates complementary sense gypsy transcripts in somatic cells of the Drosophila ovary. J. Virol. 2007, 81, 1951-1960. [CrossRef]

10. Tan, H.; Qurashi, A.; Poidevin, M.; Nelson, D.L.; Li, H.; Jin, P. Retrotransposon activation contributes to fragile X premutation rCGG-mediated neurodegeneration. Hum. Mol. Genet. 2011, 21, 57-65. [CrossRef]

11. Andrenacci, D.; Cavaliere, V.; Lattanzi, G. The role of transposable elements activity in aging and their possible involvement in laminopathic diseases. Ageing Res. Rev. 2020, 57, 100995. [CrossRef]

12. Driver, C.J.; McKechnie, S.W. Transposable elements as a factor in the aging of Drosophila melanogaster. Ann. N. Y. Acad. Sci. 1992, 673, 83-91. [CrossRef]

13. Lattanzi, G.; Ortolani, M.; Columbaro, M.; Prencipe, S.; Mattioli, E.; Lanzarini, C.; Maraldi, N.M.; Cenni, V.; Garagnani, P.; Salvioli, S.; et al. Lamins are rapamycin targets that impact human longevity: A study in centenarians. J. Cell Sci. 2014, 127, 147-157. [CrossRef] [PubMed]

14. Wood, J.G.; Helfand, S.L. Chromatin structure and transposable elements in organismal aging. Front. Genet. 2013, 4, 274. [CrossRef]

15. Saleh, A.; Macia, A.; Muotri, A.R. Transposable Elements, Inflammation, and Neurological Disease. Front. Neurol. 2019, 10, 894. [CrossRef] [PubMed]

16. Simon, M.; Van Meter, M.; Ablaeva, J.; Ke, Z.; Gonzalez, R.S.; Taguchi, T.; De Cecco, M.; Leonova, K.I.; Kogan, V.; Helfand, S.L.; et al. LINE1 Derepression in Aged Wild-Type and SIRT6-Deficient Mice Drives Inflammation. Cell Metab. 2019, 29, 871-885.e5. [CrossRef]

17. Wood, J.G.; Jones, B.C.; Jiang, N.; Chang, C.; Hosier, S.; Wickremesinghe, P.; Garcia, M.; Hartnett, D.A.; Burhenn, L.; Neretti, N.; et al. Chromatin-modifying genetic interventions suppress age-associated transposable element activation and extend life span in Drosophila. Proc. Natl. Acad. Sci. USA 2016, 113, 11277-11282. [CrossRef]

18. Prud'homme, N.; Gans, M.; Masson, M.; Terzian, C.; Bucheton, A. Flamenco, a gene controlling the gypsy retrovirus of Drosophila melanogaster. Genetics 1995, 139, 697-711. [CrossRef] [PubMed]

19. Guida, V.; Cernilogar, F.M.; Filograna, A.; De Gregorio, R.; Ishizu, H.; Siomi, M.C.; Schotta, G.; Bellenchi, G.C.; Andrenacci, D. Production of Small Noncoding RNAs from the flamenco Locus Is Regulated by the gypsy Retrotransposon of Drosophila melanogaster. Genetics 2016, 204, 631-644. [CrossRef] [PubMed]

20. Desset, S.; Meignin, C.; Dastugue, B.; Vaury, C. COM, a heterochromatic locus governing the control of independent endogenous retroviruses from Drosophila melanogaster. Genetics 2003, 164, 501-509. [CrossRef]

21. Duc, C.; Yoth, M.; Jensen, S.; Mouniée, N.; Bergman, C.M.; Vaury, C.; Brasset, E. Trapping a somatic endogenous retrovirus into a germline piRNA cluster immunizes the germline against further invasion. Genome Biol. 2019, 20, 127. [CrossRef] [PubMed]

22. Tsybul'ko, E.A.; Morozova, T.V.; Filatov, D.A.; Pasiukova, E.G. Age dependence of the level of copia retrotransposon expression in the testes of Drosophila melanogaster. Genetika 2004, 40, 1058-1062. [CrossRef] [PubMed]

23. Li, W.; Prazak, L.; Chatterjee, N.; Grüninger, S.; Krug, L.; Theodorou, D.; Dubnau, J. Activation of transposable elements during aging and neuronal decline in Drosophila. Nat. Neurosci. 2013, 16, 529-531. [CrossRef] [PubMed] 